INPLASY

PROTOCOL

To cite: Mai et al. Effectiveness and safety of Er-xian Decoction (traditional Chinese medicine) for women with Primary ovarian insufficiency. Inplasy protocol 2021110107. doi:

10.37766/inplasy2021.11.0107

Received: 29 November 2021

Published: 29 November 2021

Corresponding author: Hongxia Ma

doctorhongxia@126.com

Author Affiliation:

The First Affiliated Hospital of

Guangzhou Medical University.

Support: National Natural

Science Found.

Review Stage at time of this submission: Preliminary

searches.

Conflicts of interest:

None declared.

\section{Effectiveness and safety of Er-xian Decoction (traditional Chinese medicine) for women with Primary ovarian insufficiency}

\author{
Mai, ZF1; Lu, CL2; Zhuang, ZX3; Ma, HX4.
}

Review question / Objective: To assess the effectiveness and safety of Er-xian Decoction in the treatment of primary ovarian insufficiency.

Information sources: We will search the following electronic databases, including 3 English databases (PubMed, EMBASE, Cochrane Library) and 4 Chinese databases (China national knowledge infrastructure database, Wanfang database, Sinomed Database, and VIP database). The filters were English and Chinese language. The following key words in Title/Abstract or MeSH search headings are used: "Er-xian" and "Hormone replacement therapy" or "Femoston" or "Climen" and "Primary ovarian insufficiency" or "Ovarian failure" or "Premature ovarian failure" or "POI" and "random"” or "Randomized controlled trial". In addition, we also search the grey literature such as conference proceedings and dissertations in CNKI and Wanfang database, and relevant trials will be searched in ClinicalTrial.gov database [20] and Chinese Clinical Trial Registry for unpublished trials and protocols. References of all included studies will be hand searched for additional eligible studies.

INPLASY registration number: This protocol was registered with the International Platform of Registered Systematic Review and Meta-Analysis Protocols (INPLASY) on 29 November 2021 and was last updated on 29 November 2021 (registration number INPLASY2021110107).

\section{INTRODUCTION}

Review question / Objective: To assess the effectiveness and safety of Er-xian Decoction in the treatment of primary ovarian insufficiency.
Condition being studied: Primary ovarian insufficiency (POI), refers to the occurrence of primary or secondary amenorrhea, low estrogen level, and high gonadotropin level in women ( $<40$ years old) [1]. European Society for Human Reproduction and Embryology (ESHRE) reported to reduce 
the diagnostic critical value of FSH to $25 \mathrm{U} /$ L in the 2016 Guideline, aiming to detect, diagnose and treat for $\mathrm{POI}$ earlier. $\mathrm{POI}$ is mainly caused by genetic, autoimmune, and environmental factors, leading to the decrease number of primordial follicle and atresia [2-3], which affect the fertility and health of women. POI are always have the symptoms such as hot flashes, night sweats, insomnia, anxiety and irregular menstruation, the clinical incidence is $1 \%$ $3 \%$ [4]. With the progression of POI, not only the patient's ovarian function will gradually fail, but also the risk of cardiovascular disease and osteoporosis will gradually increase. Hormone replacement therapy (HRT) is mainly used to mimics hormone changes in the natural menstrual cycle [2], which can inhibit the release of FSH and increase the sensitivity of follicles to endogenous $\mathrm{FSH}$, stimulate the hypothalamic pituitary gland to increase LH secretion and regulate the state of low estrogen and high gonadotropin [5]. The reconstruction of menstrual cycle and the periodic supplement of estrogen and progesterone, are conducive to the recovery of uterine function. However, HRT has limited efficacy and may not significantly restore ovarian function after withdrawal. Long-term use of hormones will increase the potential risk of cardiovascular disease, breast cancer, endometrial cancer and so on. In addition, HRT is not suitable for all POI patients because of certain contraindications such as breast tumor and endometrial cancer history [6-7]. It is reported that Traditional Chinese Medicine (TCM) can adjust the level of endocrine hormone in POI women, improve the morphology and blood circulation in ovarian tissue, regulate immunity, restore part of ovarian function, and effectively reduce the adverse reactions caused by HRT [8]. In TCM, POI belongs to the categories of "amenorrhea" and "infertility" which should be treated by the principles of tonifying kidney and filling essence, promoting blood circulation and removing clot. Er-xian Decoction (EXD) can tonify kidney and regulae Chong and Ren, which is in line with the principle of treating POI in TCM. It is reported that EXD can improve symptoms which caused by the disorder of hormone levels, strengthen the immune system, regulate the level of reproductive endocrine and promote the internal environment of the body to reach a relatively stable state. Moreover, it can regulate women's autonomic nerve function, improve a series of perimenopausal syndrome caused by the decrease of estrogen level and the increase of gonadotropin level [8-9]. Studies of animal experimental have confirmed that EXD can improve the blood supply of ovarian, restore the recruitment and maturation of follicular, reduce the atresia of follicular, inhibit the apoptosis of cell and promote the proliferation of ovarian granulosa cell so as to improve the function of ovarian [10-12]. Clinical trials have shown that EXD can effectively downregulate the level of FSH and up-regulate the level of E2 in POI patients, relieve their symptoms such as hot flashes, night sweats, insomnia and irritability, promote the recurrence of menstrual [13-15]. EXD combined with HRT has better efficacy and fewer adverse reactions than HRT alone [16-17]. There is still a lack of systematic review to summarize the curative effect of EXD and provide effective evidence for clinical practice though EXD has a good curative effect. Therefore, the purpose of this study is to systematically review current relevant randomized controlled clinical trials to evaluate the effectiveness and safety of EXD compared with HRT in the treatment of POI.

\section{METHODS}

Search strategy: 1) CNKI: (TKA=“卵巢早衰' OR TKA = “卵巢衰竭” OR TKA= ‘早发性卵巢功 能不全” OR TKA= ‘原发性卵巢功能不全”) AND (TKA= “二仙汤”) AND (TKA= “激素” OR TKA= “激素疗法” OR TKA= ‘激素替代法” OR TKA= “激素替代疗法” OR TKA= ‘激素序贯疗法” OR TKA= “激素周期疗法” OR TKA= '雌孕激素疗 法' OR TKA= '雌孕激素联合疗法' OR TKA= '人工周期疗法' OR TKA= '芬吗通' OR TKA= ‘克龄蒙') AND (TKA= ‘随机” OR TKA= ‘随机对 照试验') 2) Wanfang: (题名或关键词:(卵巢早 衰 OR 卵巢衰竭 OR 早发性卵巢功能不全 OR 
原发性卵巢功能不全) OR 摘要:(卵巢早衰 OR 卵巢衰竭 OR 早发性卵巢功能不全 OR 原发性 卵巢功能不全)) AND (题名或关键词:(二仙汤) OR 摘要:(二仙汤)) AND (题名或关键词:(激素 OR 激素疗法 OR 激素替代法 OR 激素替代疗 法 OR 激素序贯疗法 OR 激素周期疗法 OR 雌 孕激素疗法 OR 雌孕激素联合疗法 OR 人工周 期疗法 OR 克龄蒙 OR 芬吗通) OR 摘要:(激素 OR 激素疗法 OR 激素替代法 OR 激素替代疗 法 OR 激素序贯疗法 OR 激素周期疗法 OR 雌 孕激素疗法 OR 雌孕激素联合疗法 OR 人工周 期疗法 OR 克龄蒙 OR 芬吗通)) AND (题名或关 键词:(随机OR 随机对照试验) OR 摘要:(随机OR 随机对照试验)) 3) VIP：(M=( 卵巢早衰 OR 卵 巢衰竭 OR 早发性卵巢功能不全 OR 原发性卵 巢功能不全 ) OR $R=$ (卵巢早衰 $O R$ 卵巢衰竭 OR 早发性卵巢功能不全 OR 原发性卵巢功能 不全 )) AND ( $M=($ 二仙汤 ) OR $R=$ (二仙汤)) AND (M=( 激素 OR 激素疗法 OR 激素替代法 OR 激素替代疗法 OR 激素序贯疗法 OR 激素 周期疗法 OR 雌孕激素疗法 OR 䧳孕激素联合 疗法 OR 人工周期疗法 OR 克龄蒙 OR 芬吗通 ) OR R=(激素 OR 激素疗法 OR 激素替代法 OR 激素替代疗法 OR 激素序贯疗法 OR 激素周期 疗法 OR 雌孕激素疗法 OR 雌孕激素联合疗法 OR 人工周期疗法 OR 克龄蒙 OR 芬吗通 )) AND ( $M=($ 随机 OR 随机对照试验) OR $R=($ 随 机 OR 随机对照试验 )) 4) SINOMED: ((()(("卿 巢早衰"[常用字段:智能] OR "卵巢衰竭"[常用字 段:智能] OR "原发性卵巢功能不全"[常用字段: 智能] OR "早发性卵巢功能不全"[常用字段:智 能])) AND ("二仙汤"[常用字段:智能]))) AND (("激素"[常用字段:智能] OR "激素疗法"[常用字 段:智能] OR "激素替代法"[常用字段:智能] OR "激素替代疗法"[常用字段:智能] OR "激素序贯 疗法"[常用字段:智能] OR "激素周期疗法"[常用 字段:智能] OR "雌孕激素疗法"[常用字段:智能] OR "雌孕激素联合疗法"[常用字段:智能] OR "人工周期疗法"[常用字段:智能] OR "克龄 蒙"[常用字段:智能] OR "芬吗通"[常用字段:智 能]))) AND (("随机"[常用字段:智能] OR “随机 对照试验"[常用字段:智能])))
5) PUBMED: \#1:((()(()((Primary ovarian insufficiency[MeSH Terms]) OR (POF[Title/ Abstract])) OR (POI[Title/Abstract])) OR (premature*[Title/Abstract])) OR (premature ovarian failure[Title/Abstract])) OR (Resistant Ovary Syndrome[Title/Abstract])) OR (Ovarian Failure[Title/Abstract])) OR (ovarian insufficiency[Title/Abstract])) OR (Ovarian Failure, premature[Title/Abstract])) OR (ovarian insufficiency, primary[Title/ Abstract]) \#2: erxian[Title/Abstract] \#3: ()( (()((Hormone replacement therapy[MeSH Major Topic]) OR (HRT[Title/Abstract])) OR (HT[Title/Abstract])) OR (Hormone Hormone replacement[Title/Abstract])) OR (Replacement therapy[Title/Abstract])) OR (Replacement therapy,Hormone[Title/ Abstract])) OR (Therapy,Hormone replacement[Title/Abstract])) OR (Hormone sequential therapy[Title/Abstract])) OR (CIimen [Title/Abstract])) OR (Femoston[Title/Abstract]) \#4: (Randomized controlled trial[MeSH Major Topic]) OR ( $r$ a n d o m * [ T i l e / A b s t r a c t ]) \#1AND\#2AND\#3AND\#4

6)COCHRANE: \#1: Title abstract keyword: Primary ovarian insufficiency OR POF OR POI OR premature* OR premature ovarian failure OR Resistant Ovary Syndrome OR Ovarian Failure OR ovarian insufficiency OR Ovarian Failure, premature OR ovarian insufficiency, primary \#2: Title abstract keyword: Medicine, Chinese Traditional OR Drugs, Chinese Herbal OR TCM OR Chinese Medicine, Traditional OR Chinese Traditional Medicine OR Chinese medicinal formula OR Chinese drugs, plant OR Chinese herbal drugs OR Chinese drug OR Chinese medicine OR Chinese herbal medicine OR Chinese herbal remedy OR Chinese patent medicine OR Chinese patent drug OR Traditional Chinese Medicine patent OR Traditional Chinese Medicine OR Traditional Medicine, Chinese OR Herbal drugs, Chinese OR Herbal preparations OR Herbal remedy OR Herbal supplement* OR Herbal medicine OR herb* OR Proprietary Chinese medicine \#3: Title abstract keyword: Hormone replacement therapy OR HRT OR HT OR Hormone OR Hormone replacement OR Replacement therapy OR Replacement therapy, Hormone OR Therapy, Hormone 
replacement OR Hormone sequential therapy OR Climen OR Femoston \#4: Title abstract keyword: Randomized controlled trial OR random* \#5:1and2and3and4

7)EMBASE: \#1: 'premature ovarian failure'/ exp/mj OR pof:ti,ab,kw OR poi:ti,ab,kw OR premature*:ti,ab,kw OR 'premature ovarian failure':ti,ab,kw OR 'resistant ovary syndrome':ti,ab,kw OR 'ovarian failure':ti, ab, kw OR 'ovarian insufficiency':ti,ab,kw OR 'ovarian failure, premature':ti,ab,kw OR 'ovarian insufficiency, primary':ti,ab,kw \#2: 'chinese medicine'/exp/mj OR 'erxian':ti,ab,kw OR 'drugs, chinese herbal':ti,ab,kw OR tcm:ti,ab,kw OR 'chinese medicine, traditional':ti,ab,kw OR 'chinese traditional medicine':ti,ab,kw OR 'chinese medicinal formula':ti,ab,kw OR 'chinese drugs, plant':ti,ab,kw OR 'chinese herbal drugs':ti,ab,kw OR 'chinese drug':ti,ab,kw OR 'chinese medicine':ti,ab,kw OR 'chinese herbal medicine':ti,ab,kw OR 'chinese herbal remedy':ti,ab,kw OR 'chinese patent medicine':ti,ab,kw OR 'chinese patent drug':ti,ab,kw OR 'traditional chinese medicine patent':ti,ab,kw OR 'traditional chinese medicine':ti,ab,kw OR 'traditional medicine, chinese':ti,ab,kw OR 'herbal drugs, chinese':ti,ab,kw OR 'herbal preparations':ti,ab,kw OR 'herbal remedy': $t i, a b, k w$ OR ' herbal supplement*':ti,ab,kw OR 'herbal medicine':ti,ab,kw OR herb*:ti,ab,kw OR 'proprietary chinese medicine':ti,ab,kw \#3: 'hormone substitution'/exp/mj OR hrt:ti,ab,kw OR ht:ti,ab,kw OR hormone:ti, ab,kw OR 'hormone replacement':ti,ab,kw OR 'replacement therapy':ti,ab,kw OR 'replacement therapy, hormone':ti,ab,kw OR 'therapy, hormone replacement':ti,ab,kw OR 'hormone sequential therapy':ti,ab,kw OR climen:ti,ab,kw OR estradiol:ti,ab,kw \#4: 'randomized controlled trial'/exp/mj OR rct:ti,ab,kw OR random*:ti,ab,kw OR 'controlled trial, randomized':ti,ab,kw OR 'randomised controlled study':ti,ab,kw OR 'randomised controlled trial':ti,ab,kw OR 'randomized controlled study':ti,ab,kw OR 'randomized controlled trial':ti,ab,kw \#5:1and2and3and4.
8) ClinicalTrial.gov database: Status: All studies Condition or disease: Primary ovarian insufficiency Other terms: Erxian

9) Chinese Clinical Trial Registry for unpublished trials and protocols:

注册状态：不限

研究所处阶段：不限

研究设计：随机平行对照

研究类型：干预性研究

研究疾病名称：早发性卵巢功能不全

干预措施：二仙汤

Participant or population: Women diagnosed with POI by defined criteria base on the European Society for Human Reproduction and Embryology (ESHRE) Guideline: a) 25 IU/I on two occasions $>4$ weeks apart. In addition, women whose FSH level $>40 \quad$ IU/I and diagnose with Premature ovarian failure are also included $[2,7]$.

Intervention: Er-xian Decoction used alone or in combination with Hormone therapies for women ( $<40$ years) were included.

Comparator: The control group were treated with Hormone replacement therapy (HRT).

Study designs to be included: Randomized controlled trial.

Eligibility criteria: Inclusion criteria: 1)Types of studies:Randomized controlled trial. 2)Types of participants:Women diagnosed with POI by defined criteria base on the European Society for Human Reproduction and Embryology (ESHRE) Guideline: a) 25 IU/I on two occasions $>4$ weeks apart. In addition, women whose FSH level $>40$ IU/I and diagnose with Premature ovarian failure are also included [2,7]. 3)Types of interventions [2,6-9]:Er-xian Decoction used alone or in combination with Hormone therapies for women ( $<40$ years) were included. Er-xian Decoction consist of Curculiginis Rhizome (Xianmao, 15g), Epimrdii Herba (Yinyanghuo, 15g), Angelicae Sinensis Radix (Danggui, 9g), Morindae Officinalis Radix (Bajitian, 9g), 
Phellodendri Chinrnsis Cortex (Huangbo, 9g), and Anemarrhenae Rhizoma (Zhimu, 9g). The experimental group were treated with Er-xian Decoction alone or combined with Hormone therapy. Er-xian Decoction can be plus or minus herbs on the basis of patients. The control group were treated with HRT. The most common EstrogenProgesterone cycle therapy was taking estrogen tablets continuously for 21 days and adding progesterone tablets from day 12 to day 21 . Climen was used for every 21 days as a course, 1 estrogen tablet was taken daily on the first 11 days and 1 compound tablet which consisted of estrogen and progesterone was taken daily on the last 10 days. Femoston was used for every 28 days as a course of treatment, on the first 14 days, 1 estrogen tablet was taken daily, on the last 14 days, 1 compound tablet which consisted of estrogen and progesterone was taken daily. 4)Types of outcomes[2、7-8]: a)Primary outcomes: a1)The levels of following hormones:FSH, LH,E2; a2)Menstruation situations, which were measured by cycles, periods and menstrual volumes. b) Secondary outcomes: b1)Clinical symptoms: hot flashes, night sweats, insomnia, mood etc. (Clinical symptoms were objectively evaluated by using Kupperman Scale and Menopause Rating Scale); b2)Adversereactions. Exclusion criteria: 1)Participants with other diseases (breast tumors, endometrial cancer, cardiovascular disease, thyroid dysfunction, hyperprolactinemia, primary hypogonadism); 2) Participants with genital malformation, or amenorrhea caused by chemotherapy drugs (such as cisplatin, cyclophosphamide, epirubicin, etc.), surgery, infection and other factors; 3)Participants with amenorrhea caused by other ovulatory dysfunction diseases (polycystic ovary syndrome, ovarian resistance syndrome and low ovarian reserve); 4) The experimental group or control group combined with other treatment methods (such as acupuncture, moxibustion, other non-symptomatic drugs); 5) Duplicate publication (Determine whether a randomized controlled trial was the same study based on the basic information of study location, study time, investigator, and funding support. The later articles should be excluded from repetitive publications, and articles with different outcome indicators from the same study should be included and analyzed as the same study).

Information sources: We will search the following electronic databases, including 3 English databases (PubMed, EMBASE, Cochrane Library) and 4 Chinese databases (China national knowledge infrastructure database, Wanfang database, Sinomed Database, and VIP database). The filters were English and Chinese language. The following key words in Title/Abstract or MeSH search headings are used: "Er-xian" and "Hormone replacement therapy" or "Femoston" or "Climen" and "Primary ovarian insufficiency" or "Ovarian failure" or "Premature ovarian failure" or "POI" and "random"" or "Randomized controlled trial". In addition, we also search the grey literature such as conference proceedings and dissertations in CNKI and Wanfang database, and relevant trials will be searched in ClinicalTrial.gov database [20] and Chinese Clinical Trial Registry for unpublished trials and protocols. References of all included studies will be hand searched for additional eligible studies.

Main outcome(s): 1)The levels of following hormones: FSH; LH; E2 2) Menstruation situations, which were measured by cycles, periods and menstrual volumes.[2, 7-8].

Additional outcome(s): 1) Clinical symptoms: hot flashes, night sweats, insomnia, mood etc. (Clinical symptoms were objectively evaluated by using Kupperman Scale and Menopause Rating Scale) 2) Adverse reactions [2, 7-8].

Data management: Two authors (Zhefen Mai and Zixun Zhuang) will screen all studies independently by Endnote software to identify the eligible trials and extracted the following data independently: first author, year of publication, study design, 
sample sizes, diagnosis criteria, inclusion and exclusion criteria, interventions, the reported outcome and adverse events. Different opinions will be discussed and resolved through consulting the third author (Chunli Lu). We will use RevMan 5.4 software for data analysis. The dichotomous variables (counting data) will be described by Relative Risk (RR) with 95\% Confidence Intervals $(95 \% \mathrm{Cl})$, and the continuous variables (measurement data) will be expressed by mean difference (MD) with $95 \%$ Confidence Intervals $(95 \% \mathrm{Cl})$. We performed meta-analyses for trials if they are similar in the study design, participants, interventions, control, and outcome measures. Qualitative description and analysis will be used if the data cannot be combined and analyzed.

Quality assessment / Risk of bias analysis: Two authors (Zhefen Mai and Zixun Zhuang) will use the Cochrane Collaboration tool for Assessing risk of bias in randomized trial to assess the quality of the included trials [18]. The trials were appraised as "High risk," "Low risk," or "Unclear" by the following 7 items: random sequence generation, allocation concealment, blinding of participants and personnel, blinding of outcome assessment, incomplete outcome data, selective reporting and other bias such as sample size and pharmaceutical funding. Any disagreements were discussed and resolved with the third author (Chunli Lu).

Strategy of data synthesis: We will use RevMan 5.4 software for data analysis. The dichotomous variables (counting data) will be described by Relative Risk (RR) with $95 \%$ Confidence Intervals $(95 \% \mathrm{Cl})$, and the continuous variables (measurement data) will be expressed by mean difference (MD) with $95 \%$ Confidence Intervals $(95 \% \mathrm{Cl})$. We performed meta-analyses for trials if they are similar in the study design, participants, interventions, control, and outcome measures. Qualitative description and analysis will be used if the data cannot be combined and analyzed.

Subgroup analysis: If the data included in the trial are available like BMI grouping or
Age groups [19], the subgroup analysis will be performed.

Sensitivity analysis: The heterogeneity of included trials will be judged by I-square. We consider the heterogeneity of trials are obvious if $12>50 \%$ and random effects model (REM) should be used for analyzing. Otherwise, a fixed effects model (FEM) will be used. Data analysis results will be represented by forest map and publication bias will be represented by funnel plot.

Language: Chinese and English.

Country(ies) involved: China.

Other relevant information: Stage 1) September 01, 2021 to October 30, 2021: The retrieval, screening and evaluation of literature; 2) November 01, 2021 to December 30, 2021: Data extraction and analysis; 3) January 01, 2022 to January 31, 2022: Writing meta-analysis.

Author Affiliation:

Miss Zhefen Mai. The First Affiliated Hospital of Guangzhou Medical University Ph.D Chunli Lu. Centre for Evidence-Based Chinese Medicine, Beijing University of Chinese Medicine

Miss Zixun Zhuang. The First Affiliated Hospital of Guangzhou Medical University Professor Hongxia Ma. The First Affiliated Hospital of Guangzhou Medical University Zhefen Mai, Chunli Lu and Zixun Zhuang share first authorship.

Keywords: Primary ovarian insufficiency; Er-xian; Hormone replacement therapy.

Contributions of each author:

Author 1 - Zhefen Mai.

Email: maizhefen@126.com

Author 2 - Chunli Lu.

Email: jennylu@bucm.edu.cn

Author 3 - Zixun Zhuang.

Email: 516535540@qq.com

Author 4 - Hongxia Ma.

Email: doctorhongxia@126.com

References: [1] Collins G, Patel B, Thakore S, Liu J. Primary Ovarian Insufficiency: Current Concepts. South Med J. 2017 
Mar;110(3):147-153. doi: 10.14423/ SMJ.0000000000000611. PMID: 28257537. [2] European Society for Human Reproduction and Embryology (ESHRE) Guideline Group on POI, Webber L, Davies $M$, Anderson R, et al. ESHRE Guideline: management of women with premature ovarian insufficiency. Hum Reprod. 2016 May;31(5):926-37. doi: 10.1093/humrep/ dew027. Epub 2016 Mar 22. PMID: 27008889. [3] Maclaran K, Panay N. Current concepts in premature ovarian insufficiency. Womens Health (Lond). 2015 Mar;11(2):169-82. doi: 10.2217/whe.14.82. PMID: 25776291. [4] Committee on Gynecologic Practice. Committee opinion No. 698: hormone therapy in primary ovarian insufficiency[J]. Obstet Gynecol, 2017, 129( 5) : e134-e141. [5]CAO Jinxiang, WU Jie. Evaluation of benefits and risks of hormone replacement therapy for the women with premature ovarian insufficiency. Chinese Journal of Practical Gynecology and Obstetrics. 2020(03):227-230. doi:10.19538/ j.fk2020030111. [6]LI Min, GUO Miao, SUN Jianhua, et al. Network Pharmacological Analysis of Erxian decoction in the treatment of POI. World Chinese Medicine. (Accepted manuscript) Online First Publishing Date: 2021-05-27 14:46:08. [7] CHEN Zijiang, TIAN Qinjie, QIAO Jie, et al. Chinese expert consensus on the clinical diagnosis and treatment of primary ovarian insufficiency. Chinese Journal of Gynecology and Obstetrics. 2017,52(09):577-581. [8]ZENG Huo, CHEN Qiuhong. Efficacy analysis of Erxian decoction for improving symptoms and endocrine indexes of patients with premature ovarian failure. Clinical Journal of Chinese Medicine. 2017(03):87-88. [9]YANG Lin, MA Hong. Review of Erxian Decoction on Gynecological Clinical Application and Experimental Research. Journal of Liaoning University of Traditional Chinese Medicine. 2016(10):94-97. doi:10.13194/j.issn.1673-842x.2016.10.028. [10]YANG Lei, WANG Jifeng. Experimental research progress of Erxian decoction and its disassembled prescriptions in treatment of premature ovarian failure. Global Traditional Chinese Medicine.
2017(05):626-630. [11]MAO Xianglin, BAO Weiqian, ZHAO Piwen. Influence of Erxian Decoction on ovarian reserve of rats suffered from premature ovarian failure. China Journal of Traditional Chinese Medicine and Pharmacy. 2017(02):771-773. [12]ZHAO Di, ZHAO Piwen, WU Hongbo. Influences of Erxian decoction on proliferation and cell cycle of ovarian granulosa cells in premature ovarian failure model caused by CDDP. Global Traditional Chinese Medicine. 2017(02):131-136. [13]YUAN Yingjun, WANG Xiaoyan, LAN Lan. Observation on the Therapeutic Effect of Erxian Decoction on Early-onset Ovarian Insufficiency of Spleen and Kidney Yang Deficiency Type. Systems Medicine. 2019(18): 130-132. doi:10.19368/ j.cnki.2096-1782.2019.18.130. [14] Jia Chengjun. Observation on curative effect of treating premature ovarian failure amenorrhea with kidney deficiency and Liver depression by Erxian Decoction. Journal of China Prescription Drug. 2019(05):104-105. [15] Zhang Hongyan, LI Ling, GUO Ruilian. Observation on curative effect of treating premature ovarian failure amenorrhea with kidney deficiency and Liver depression by Erxian Decoction. Journal of Practical Traditional Chinese Medicine. 2018(04):419-420. [16]LIN Xia, CHEN Hua, CAI Yuping, et al. Clinical Study on Bushen Erxian Tang Combined with Estrogen and Progesterone for Premature Ovarian Failure. Journal of New Chinese Medicine. 2019(07):170-172. doi:10.13457/ j.cnki.jncm.2019.07.050. [17] Yi Zixuan. Clinical efficacy of Erxian Decoction combined with estrogen progesterone sequential therapy in treatment of premature ovarian failure with Deficiency of Yin and Yang. Electronic Journal of General Stomatology. $2020(01): 164-165$. doi:10.16269/j.cnki.cn11-9337/ r.2020.01.123. [18]Higgins JPT, Thomas J, Chandler J, Cumpston M, Li T, Page MJ, Welch VA (editors). Cochrane Handbook for Systematic Reviews of Interventions version 6.2 (updated February 2021). Cochrane, 2021. Available from http:// www.training.cochrane.org/handbook. [19]Buyuk E, Seifer DB, Illion E, et al. Elevated body mass index is associated 
with lower serum anti-mullerian hormone levels in infertile women with diminished ovarian reserve but not with normal ovarian reserve [J]. Fertil Steril, 2011, 95: 2364-2368. [20]Wu Dan, Chang Feng, Shao Rong. The Research on Construction of Information and Retrieval Service of the US Clinical Trial Database. China Licensed Pharmacist. 2011(04) :24-27. 